\title{
Current blockage and extreme forces on a jacket model in focussed wave groups with current
}

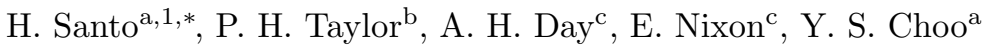 \\ ${ }^{a}$ Centre for Offshore Research $\& 6$ Engineering, Department of Civil and Environmental Engineering, National \\ University of Singapore, Singapore 117576, Singapore \\ ${ }^{b}$ Department of Engineering Science, University of Oxford, Oxford OX1 3PJ, United Kingdom \\ ${ }^{c}$ Department of Naval Architecture, Ocean and Marine Engineering, University of Strathclyde, Glasgow G4 OLZ, \\ United Kingdom
}

\begin{abstract}
This paper documents large laboratory-scale measurements of hydrodynamic force time histories on a realistic 1:80 scale space-frame jacket structure exposed to combined waves and in-line current. The aim is to investigate the fluid flow (and the associated hydrodynamic force) reduction relative to ambient fluid flow due to the presence of the jacket structure as an obstacle array, interpreted as wave-current blockage. Transient focussed wave groups, and embedded wave groups in a smaller regular wave background are generated in a towing tank, and the jacket is towed under different speeds opposite to the wave direction to simulate wave loading with different in-line uniform currents. The measurements are compared with numerical predictions using Computational Fluid Dynamics (CFD), with the actual jacket represented in a three-dimensional numerical wave tank as a porous tower modelled as a uniformly distributed Morison stress field. Good agreement is achieved, both in terms of incident surface elevation as well as total force time histories, all using a single set of Morison drag $\left(C_{d}\right)$ and inertia $\left(C_{m}\right)$ coefficients. Substantial force reduction is observed under transient large crest relative to prediction from the present industry design guideline with the same Morison coefficients. We demonstrate the generality of our findings: without influence of Keulegen-Carpenter (KC) number effect, a single invariant set of $C_{d}$ and $C_{m}$ is all that is required to numerically explain and reproduce the measured total force time histories on a realistic jacket model for a large range of wave heights and non-zero current speeds.
\end{abstract}

\footnotetext{
${ }^{*}$ Corresponding author. Tel: +65 6516 6853. Fax: +65 67791635

Email address: dprharr@nus.edu.sg (H. Santo)

${ }^{1}$ Present address: Office of the Deputy President (Research and Technology), National University of Singapore, Singapore 119077, Singapore
} 
Keywords: Morison fluid loading, wave-current blockage, porous block simulation

\section{Introduction}

The hydrodynamic forces on a single cylinder and arrays of cylinders have been studied extensively in the past. Many studies have considered periodic waves only without current (or periodic oscillation without steady flow), whilst some examined the effect of waves and steady current simultaneously, see e.g. Sarpkaya and Isaacson (1981), Sarpkaya et al. (1984), Heideman and Sarpkaya (1985), Rodenbusch and Källström (1986), Allender and Petrauskas (1987), Reed et al. (1990), and Chaplin et al. (1992). Large scatter in the Morison drag and inertia coefficients $\left(C_{d}\right.$ and $\left.C_{m}\right)$ is observed, and no general conclusions have been drawn, because of the complexity of the problem due to the presence of a current interacting with waves and the structure. In this paper, we propose that a solution to the problem can be obtained by looking at realistic flow around a geometrically complicated space-frame jacket model; at least in terms of the overall loads on the entire structure. This model is a realistic representation of a typical oil and gas production platform for intermediate water depth and harsh ocean environment. It is made of multiple cylinders arranged in different orientations, and it is subjected to transient wave groups and regular waves, all with steady uniform current present.

It should be noted that the estimation of loads on space-frame structures as a topic has not 17 been an active area of research over the last few decades. Allender and Petrauskas (1987) measured 18 the peak forces on a complete $3 \mathrm{~m}$ high model of a Gulf of Mexico platform in regular waves 19 and current in a very large wave tank. They observed what we interpret as significant wave20 current blockage for a wide range of regular wave heights and steady tow speeds. In terms of the ${ }_{21}$ standard design methodology (see API 2000), they reported the necessity to use a lower value for ${ }_{22}$ the Morison drag coefficient $C_{d}$ of $0.7-0.8$ to fit the measured peak forces for waves with in-line 23 current. In contrast, a $C_{d}$ of $1.3-1.6$ was required for regular waves with no current. These 24 important but apparently little known observations prompted us to re-visit the whole problem of ${ }_{25}$ the hydrodynamics of flow through space-frame structures. Whilst framed in terms of fixed jackets, 26 this flow-structure interaction problem is obviously relevant to deepwater compliant towers, jack-up ${ }_{27}$ rigs and, most recently of practical importance, the lightweight space-frames being used to support 28 large offshore wind turbines. 
This paper extends our research of current blockage on statically-responding (fixed bottomfounded) offshore structures. The presence of such structures can be treated as obstacle arrays, which provide resistance to the incident wave and current flow on the structures. Hence, reduction in the flow and the associated hydrodynamic force is observed. This phenomenon has been reported as current blockage. The first provision to the standard offshore industry design codes, such as API (American Petroleum Institute, 2000), was due to the work by Taylor (1991), which improves the Morison equation (Morison et al., 1950). This accounts only for flow reduction due to steady current flow. Recent studies by Taylor et al. (2013) analytically demonstrated the additional flow reduction from regular waves on top of steady current, and this has been validated extensively in both experiments as well as numerical simulations using Computational Fluid Dynamics (CFD), see Santo et al. (2014a,b, 2015, 2017).

Moving on from an idealised regular wave which is simply periodic in form, we consider in this paper the effect of transient and non-periodic waves which are more representative of large waves on the open sea. To model the transient effect, we consider focussed wave groups, and to account for the presence of large waves in an on-average smaller sea-state, we embed these focussed wave groups within a smaller regular wave background. We then examine the total force time histories on a realistic jacket model obtained from laboratory-scale measurements conducted in a large towing tank. We also assess and compare the force time histories from CFD results, with the actual jacket represented in a three-dimensional numerical wave tank as a porous tower modelled as a uniformly distributed Morison stress field. We also compare the predictions using the present API recommended practice and our novel porous tower modelling approach which accounts for wavecurrent blockage effects, all with the measurements taken as the reference. For force prediction, the industry approach in the past required calibrating the Morison $C_{d}$ and $C_{m}$ using the open sea fluid kinematics without otherwise taking into account the presence of the structure. The present industry approach (such as API) has started to account for the presence of the structure due to steady flow (current blockage effects), but not the complete wave-current blockage effects. We will show that this present approach is incapable of producing the experimentally measured force time histories, and in general will result in a scatter in $C_{d}$. On the other hand, using our proposed approach, the complete measured force time histories for almost all cases with current can be reproduced using a single and consistent set of $C_{d}$ and $C_{m}$. 

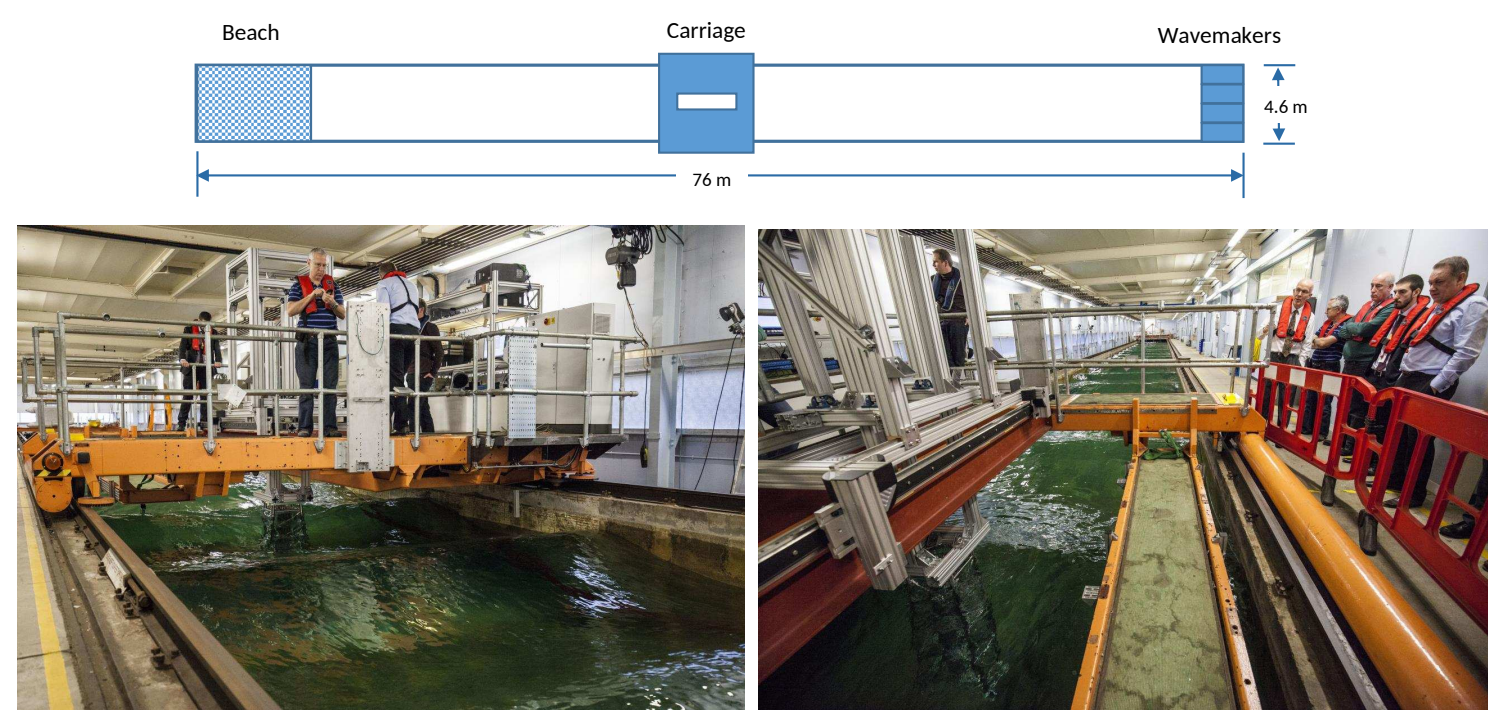

Figure 1: Top panel shows the plan view of the towing tank facility (not to scale). Bottom panel shows two photographs of the tank. Left photograph depicts the carriage with the jacket model underneath when viewed in a downstream direction along the tank where a regular wave train is incident onto the jacket model. Right photograph shows a closer look at the carriage and the jacket model when viewed in an upstream direction along the tank. Photographs courtesy of Y. S. Choo.

\section{Experimental and numerical setup}

These experiments were conducted in the towing tank of the Kelvin Hydrodynamics Laboratory, University of Strathclyde, Glasgow. This is $76 \mathrm{~m}$ long, $4.6 \mathrm{~m}$ wide and $2.5 \mathrm{~m}$ deep. The tank is equipped with four paddles of Edinburgh Design Limited (EDL) 'flap-type' wavemakers with forcefeedback at one end, and a sloping beach acting as a passive absorber at the other end. In the experiments, linear wave generation was used. A self-propelled carriage runs along the longitudinal direction of the tank. Figure 1 shows a plan view as well as two photographs of the towing tank facility.

A 1:80 jacket model was hung below the carriage, which was moved at constant speed along the tank to simulate uniform current, and the model was exposed to a range of focussed wave groups. Figure 2 shows a photograph of the jacket model with three of the authors (left), and a 3D CAD model of the jacket with relevant geometric information (right). Being made of stainless steel, the jacket model resembles a typical second generation North Sea 4-legged jacket structure. It stands at $1.74 \mathrm{~m}$ tall and weighs around $50 \mathrm{~kg}$ in the air. The cross-section of the jacket at the top is 0.39 

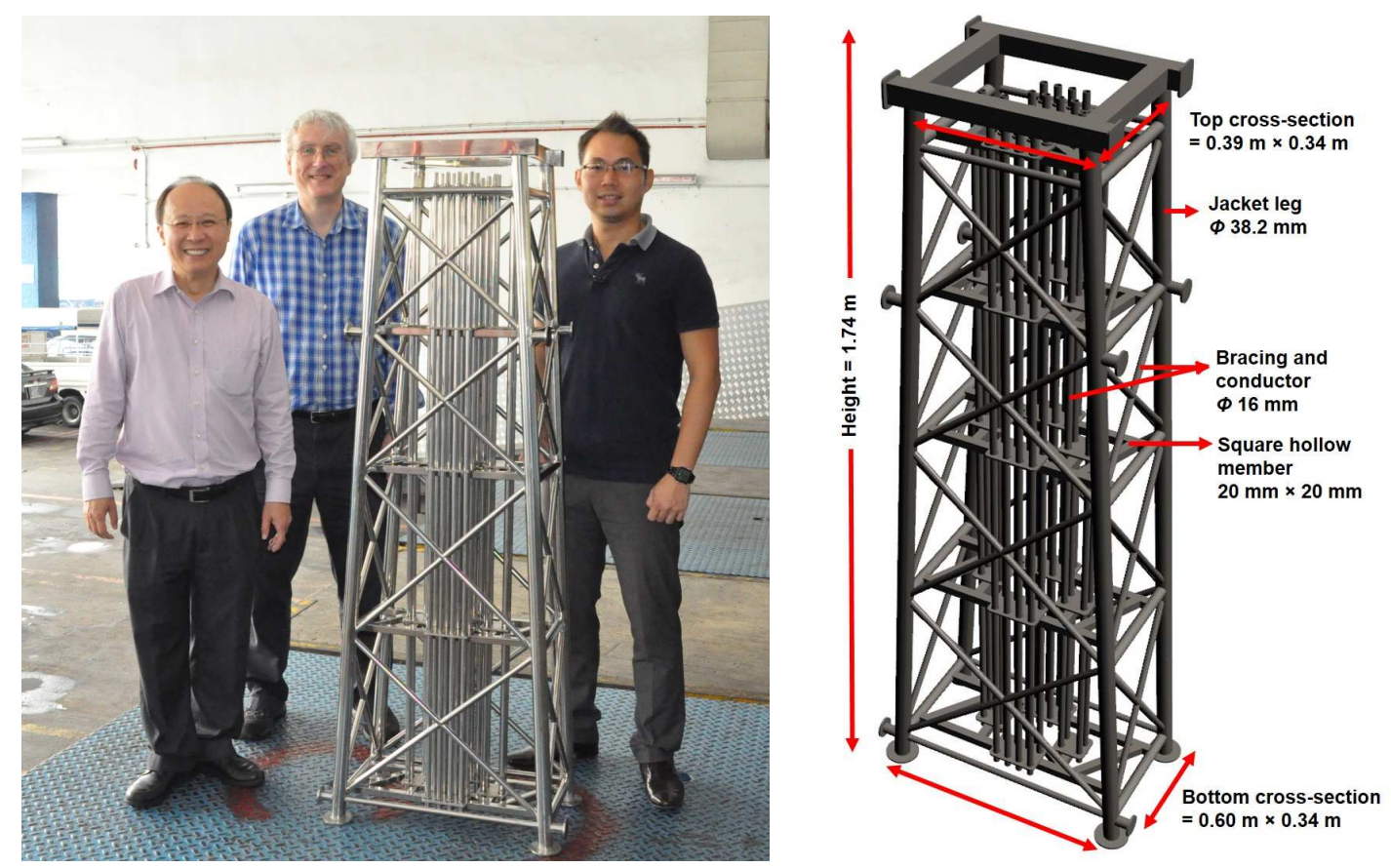

Figure 2: Left photograph shows the jacket model with three of the authors. Right picture shows a 3D CAD model of the jacket with relevant geometric information.

$\mathrm{m} \times 0.34 \mathrm{~m}$, and at the bottom is $0.60 \mathrm{~m} \times 0.34 \mathrm{~m}$. The jacket is tapered when viewed end-on and rectangular broadside. Four large cylindrical hollow members (or pipes) with a diameter of $38.2 \mathrm{~mm}$

75 (1.5") form the jacket legs. Additional smaller pipes with a diameter of $16 \mathrm{~mm}$ form the diagonal ${ }_{76}$ bracings and the vertical conductor pipes, with 24 conductors in total. These run the full height 77 of the jacket. Square hollow members with cross section of $20 \mathrm{~mm} \times 20 \mathrm{~mm}$ are used as conductor support frames at each horizontal level. These are supported on horizontal bracings at each end-on face of the jacket instead of extending from the jacket legs within the jacket (as commonly found ${ }_{80}$ in actual offshore jackets) to ease the model fabrication process. In these experiments, only the 81 end-on configuration was tested, as this will provide more blockage and a more severe test of the 82 modelling.

83 The jacket was suspended from the carriage such that the still water level is at $0.12 \mathrm{~m}$ below ${ }_{84}$ the centre of the top X-brace, or a distance of $1.33 \mathrm{~m}$ up from the jacket base. This is necessary to ensure the largest crest do not hit the top support frame. The water depth in the tank was $1.8 \mathrm{~m}$, so 86 there was a gap of $0.47 \mathrm{~m}$ between the base of the jacket model and the floor of the tank. The jacket 
model was suspended so that the total horizontal hydrodynamic load could be measured directly by a force transducer, eliminating any ambiguity in the horizontal forces since any bending moments are taken directly by the vertical elements of the support frame. This high quality measurement is made possible by having a parallel pendulum arrangement (or inverted table) for the mounting frame connected to the jacket model, and this was hung below the rigid frame attached on the towing carriage. The force transducer was rated at $50 \mathrm{~kg}(490 \mathrm{~N})$ and sampled at $7143 \mathrm{~Hz}$. A resistance-based wave probe, sampled at the same rate as the force transducer, was mounted from the towing carriage midway between the jacket model and the side of the tank to provide phase information of the incident waves.

A set of 43 Fourier wave components was generated at the paddles according to a JONSWAPshaped amplitude spectrum truncated at $1 \mathrm{~Hz}$, with the frequency of the peak spectral energy at $0.52 \mathrm{~Hz}$ and a linear crest amplitude of $0.22 \mathrm{~m}$ at focus. The water depth was set at $1.8 \mathrm{~m}$. Downstream in the tank, the wave group was arranged to focus when the crests of the Fourier components all came into phase at a single position in space and time (constructive interference). As well as an isolated focussed wave group, an embedded focussed wave within a smaller regular wave background was also considered, see for instance Figure 6 (bottom left). This is intended to model an extreme wave packet within an irregular wave sea-state, since the largest wave in a random sea is likely to be a member of a group of large waves. Regular waves were used as the background in this experiment since wave-current blockage in regular waves has been examined previously, see e.g. Santo et al. $(2015,2017)$. Three sets of regular wave with wave heights of $0.1 \mathrm{~m}, 0.13 \mathrm{~m}$ and $0.15 \mathrm{~m}$ were used for the embedding process, all with wave frequency at $0.52 \mathrm{~Hz}$. The same focussed wave components were used, but with reduced linear amplitude depending on the height of the regular wave background such that the amplitude of the embedded wave group matches that of the focussed wave group $(0.22 \mathrm{~m})$, at least on the basis of linear superposition. In the tank, the embedded wave groups interacted with the background wave and the actual focus location was shifted downstream, as consistent with Figure 7. Hence, with the background present, the embedded wave groups were not perfectly focussed (defined as having a horizontal symmetry between the adjacent troughs either side of the largest crest in time). This does not present significant difficulties for the comparison between the physical experimental forces and the CFD predictions, as iteration was used to ensure a good match between the measured and predicted incident waves at the model.

Measurements were conducted for three different towing speeds: 0, 0.14 and $0.28 \mathrm{~m} / \mathrm{s}$. Synchro- 

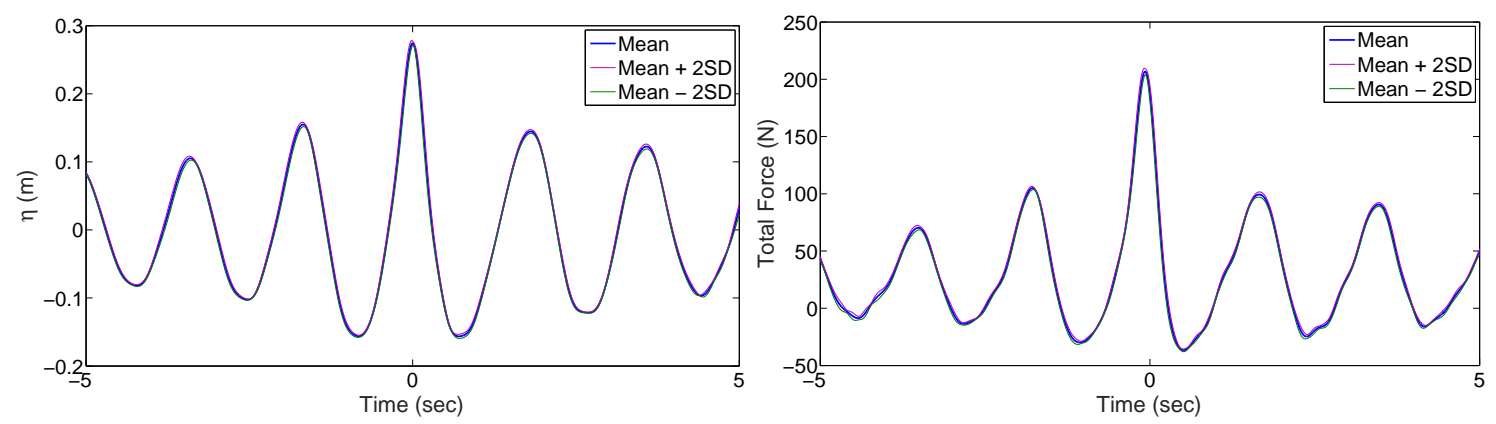

Figure 3: Comparison of repeatability in the measurement of surface elevation (left) and total force (right) time histories, all plotted in terms of mean and mean \pm 2 standard deviations.

nisation between the wave paddle and the carriage motion was carefully accounted for to ensure that the jacket model towed under different speeds meets the same wave group at the right place and at the right time. Five repeated tests were conducted for selected cases to quantify the repeatability of the system. Figure 3 presents an example of the case of $180^{\circ}$ phase-shifted embedded focussed wave in $0.15 \mathrm{~m}$ regular wave background with $0.28 \mathrm{~m} / \mathrm{s}$ current. The measurements of surface elevation are plotted on the left and total force on the right. Analysis on repeatability reveals that the root mean square error for surface elevation is $0.2 \mathrm{~cm}$ (with a peak value of $27 \mathrm{~cm}$ ), and that for total force is $1.6 \mathrm{~N}$ (with a peak of $207 \mathrm{~N}$ ), suggesting that the measurements are repeatable.

The numerical setup is similar to that reported by Santo et al. (2015) and Santo et al. (2017), using the same porous tower modelling approach with uniformly distributed embedded Morison stresses. In essence, the stresses are distributed over the tower but expressed using the local (disturbed) flow kinematics, thus accounting for the global presence of the structure. Similar work has been conducted on characterising resistance based on drag and Morison equations in related fields, see e.g. Kristiansen and Faltinsen (2012), Zhao et al. (2013), and Chen and Christensen (2016).

The simulations were performed with the open source CFD code OpenFOAM ${ }^{\circledR}$ (http://www.openfoam.com) and the numerical wave tank formulation 'waves2Foam' developed by Jacobsen et al. (2012). All the simulations are performed in two-phase flow (air and water) by solving the Reynolds-averaged Navier-Stokes equations coupled with the continuity equation for incompressible flows, and with an additional momentum sink term to account for the effect of the porous tower in the numerical 
simulation. The governing equations are written as:

$$
\nabla \cdot \mathbf{u}=0
$$

139

$$
\frac{\partial \rho \mathbf{u}}{\partial t}+\nabla \cdot\left[\rho \mathbf{u} \mathbf{u}^{T}\right]=-\nabla p^{*}+\nabla \cdot[\mu \nabla \mathbf{u}+\rho \boldsymbol{\tau}]-\mathbf{S}+\left[-(\mathbf{g} \cdot \mathbf{x}) \nabla \rho+\sigma_{T} \kappa_{\gamma} \nabla \gamma\right]
$$

140

where $\rho$ is the fluid density, $\mathbf{g}$ is the acceleration due to gravity, $\mathbf{u}=(u, v, w)$ is the fluid velocity field in Cartesian coordinates, $p^{*}$ is the pressure in excess of hydrostatic pressure, defined as $p^{*}=$ $p-(\mathbf{g} \cdot \mathbf{x}) \rho, \mu$ is the dynamic viscosity, $\mathbf{x}=(x, y, z)$ is the local Cartesian coordinates, and $\boldsymbol{\tau}$ is the specific Reynolds stress tensor. The free surface (interface between air and water) is tracked using Volume-of-Fluid (VOF) method, with an interface value ranging from 0 to 1 (0 for pure air, 1 for pure water, and a mixture in between). In the numerical simulation, the interface value of 0.5 and greater is treated as the water phase. For more details of the interface treatment, see Berberović et al. (2009).

A sink term is used to account for momentum lost from the flow, which in the case of a simple homogeneous porous tower can be written as:

$$
\mathbf{S}=\frac{1}{2} \rho F \mathbf{u}|\mathbf{u}|+C_{m}^{\prime} \frac{\partial \rho \mathbf{u}}{\partial t}
$$

where $F$ is the Forchheimer resistance parameter and $C_{m}^{\prime}$ is the equivalent of the local Morison inertia coefficient, $C_{m}$, but here defined in the porous tower context.

A porous tower having the same physical dimensions, the amount of resistance and the added mass of the actual jacket is modelled. Following Santo et al. (2014a, 2015), the following relationship holds for the calibration of $F$ and $C_{m}^{\prime}: C_{d} A / A_{f}=F L$, and $C_{m}^{\prime}=C_{m} V / V_{P}$, where $A$ and $A_{f}$ are the solid drag area and the frontal area of the actual jacket model, respectively, $L$ is the downstream length of the jacket model as well as the porous tower, $V$ is the displaced volume of the elements in the jacket model, and $V_{P}=A_{f} \times L$ is the volume of the porous tower. $C_{m}$ is the Morison inertia coefficient, and $C_{d}$ is the drag coefficient. The actual total values of $A, A_{f}$ and $V$ compatible for use in the standard Morison formulation are $1.17 \mathrm{~m}^{2}, 0.57 \mathrm{~m}^{2}$, and $0.024 \mathrm{~m}^{3}$, respectively, all measured from the bottom of the model up to $0.25 \mathrm{~m}$ above still water level. Hence, both $L$ and $V_{p}$ of the porous tower are based on the actual geometry of the jacket model. The entire resistance of the jacket is vertically split into two blocks of uniformly distributed resistance in the numerical porous tower (with a smaller block around the free-surface spanning from the largest crest to the 
deepest trough, and a larger block stacked underneath). The sensitivity of the resulting forces to such an arrangement was checked by comparing the results to those for three blocks of resistance. The integrated total force on the tower is very similar, suggesting that the arrangement is robust.

One-way information transfer (coupling) was developed to enable the full 3D simulations of focussed wave interacting with the porous tower to run using a reasonable amount of computing resource, otherwise simulating the entire 3D domain would be much more computationally expensive. The flow kinematics upstream of the focus location was sampled at small time intervals from a $2 \mathrm{D}$ simulation of undisturbed kinematics (2D in a vertical plane, so without the presence of the porous tower), and this was subsequently fed into a truncated 3D domain simulation of disturbed kinematics (with the porous tower in place). Linear interpolation was used for the flow kinematics between each sampled time interval. The truncated domain has the same width and depth as the physical towing tank, but the length is limited to $4 \lambda_{p}$, inclusive of $1 \lambda_{p}$ for the outlet relaxation zone to minimise wave reflection, where $\lambda_{p}$ is the wavelength at peak wavenumber $(\sim 5.5 \mathrm{~m})$. The sensitivity of the results to this distance was investigated by a comparison with those from a domain with length of $5 \lambda_{p}$ inclusive of $2 \lambda_{p}$ for outlet relaxation, and the same surface elevation profiles around the location of the tower are obtained. A mesh resolution of $0.025 \mathrm{~m} \times 0.025 \mathrm{~m}$ (longitudinal $\times$ vertical) is maintained around still water level which contains most of the wave action, in a similar setup as outlined in Santo et al. (2015). On average, each 3D simulation comprising 5.2 million cells took $\sim 10$ days for a 30 sec run on 12 processors. All simulations were run on the High Performance Computing (HPC) facilities of the National University of Singapore.

\subsection{Discussion on the two modelling approaches}

In this subsection, we emphasise on the key similarities and differences between the physical experiments and the numerical simulations. Using the assumption of separation of length scales, we assume that the global large-scale wake structure (which scales as the frontal width of the actual jacket) is more dominant and hence more important than the local small-scale details of the wakes for each cylinder (which scale as the diameter of the individual cylinders). Hence, we can effectively represent the actual complicated jacket model as a porous block in the numerical simulation, and calibrate the numerical model to have comparable amount of resistance and added mass as the actual model. Thus, the bulk and large-scale flow parameters are maintained going from physical to numerical model, but inevitably there are some important differences. 
The wave and current conditions in the physical experiments were reproduced as accurately as possible in the numerical wave tank. Linear wave theory was used at the numerical inlet boundary, consistent with the linear wave generation used in the physical experiments. However, instead of moving the porous tower to numerically mimic the towing of the jacket in the physical experiments, a uniform current profile was fluxed at the inlet boundary instead, mainly to avoid the additional numerical complexity of a moving (dynamic) mesh. Hence, for cases of waves with in-line current, the apparent wave encounter frequency is slightly modified in the numerical simulation to account for the Doppler shift effect introduced by the current in the physical experiments. The key difference between the two modelling approaches is thus our numerical approach has a slight effect of modifying the shape of the transient focussed wave group with uniform current relative to the same group without current. This is a result of wave-current interaction which was otherwise not present in the physical experiments, whereby the waves generated from the wave paddles at one end of the tank did not feel the effect of current. However, the aim in each of the simulations was to match both the uniform current and the wave time history at the model as accurately as possible for each physical experiment.

The possible importance of global large-scale wake interaction for total hydrodynamic load can be examined via a Keulegan-Carpenter number defined for the entire structure: $K C_{\text {struct }}=$ $2 \pi(\eta / D)$, where $\eta$ is the surface wave crest elevation and $D$ is the frontal width of the structure which is comparable to the downstream length. We base this discussion on the unsteady flow properties close to the free-surface where the wave kinematics are the largest and much of the total hydrodynamic load is exerted. For the largest waves at the centre of each wave group $\eta \sim 0.25$ $\mathrm{m}, D \sim 0.35 \mathrm{~m}$, so $K C_{\text {struct }} \sim 4.5$. For a solid body, this value of $K C$ would be small enough to exhibit very substantial $K C$ number effects. However, the volume of the space-frame model is highly porous and so the wake must be somewhat equivalent globally to that from a solid body with very considerable 'base-bleed' (Bearman, 1967) with fluid injected from the rear of the body downstream into the flow. This injected flow greatly weakens the strength of the wake, and in particular the vorticity either side of the wake. This weakened vorticity would have a smaller effect on the local flow at the porous body when swept back towards the body by the next half cycle of the wave oscillation.

For cases with both wave and inline current, we measured the force from regular waves and also a large wave group, both with a substantial current, either 0.14 or $0.28 \mathrm{~m} / \mathrm{s}$. Over the time 
required for the maximum excursion of a fluid particle to occur horizontally, here half a wave period $\sim 1 \mathrm{~s}$, the current will permanently advect global-scale vorticity downstream a distance which is a significant fraction of the whole platform width (length). Accounting for current blockage, the distances would be $\sim D / 3$ and $2 D / 3$ for the different magnitudes of the current. Hence, it is unlikely that finite $K C_{\text {struct }}$ effects would play any role, with much of the global vorticity being swept beyond the downstream edge of the structure and not returning. This would yield the same value for the drag coefficient $C_{d} \sim 1.3$ as appropriate for steady current with no waves present. The global large-scale wake interactions should be reproduced well in the numerical simulations.

The same steady flow value of $C_{d}=1.3$ can also be applied for an isolated large wave group without current and for all cases of focussed wave groups embedded into a regular wave background without current. Although a train of relatively small regular waves before an embedded large wave group would give rise to some vorticity in the flow as the large wave passes, this background wake vorticity is rather weak compared to that from the large wave itself.

Interestingly, one exception is for an incident regular wavetrain without current whereby simulation of the measured force time history requires a drag coefficient $C_{d} \sim 1.3 \times 1.6$, larger than the value of 1.3 required for the same regular waves with current or current alone. This rise from the steady-flow value of drag coefficient from 1.3 to $1.3 \times 1.6$ must be associated with $K C$ number effects, interpreted as the influence of coherently shed vortices. When a steady train of regular wave incident onto the jacket model, a group of vortices is shed forward (downstream) in one half of a regular wave cycle. The same group is subsequently swept backward (upstream) during the second half of the wave cycle. If the circulation of the previously shed vortices survives throughout the second half of the wave cycle, the flow through the gaps of the jacket model is enhanced. This would increase the hydrodynamic drag force on the structure, reflected by the requirement to increase $C_{d}$ beyond the steady flow value if the small-scale wake structure is ignored. This enhanced $C_{d}$ value in regular waves alone is of course entirely consistent with the observations of Allender and Petrauskas (1987), whose original paper provided the motivation for our blockage work.

There are no small-scale wake eddies resolved in the numerical simulations since the individual structural elements in the physical space-frame model are not represented. Instead, their global effects are being represented by a distributed stress on the fluid. This absence is clearly demonstrated in Figure 4 (left plot), where both the power spectra of the total applied force measured in the tank and force predicted by the CFD simulation are shown. The drag term in the Morison equa- 

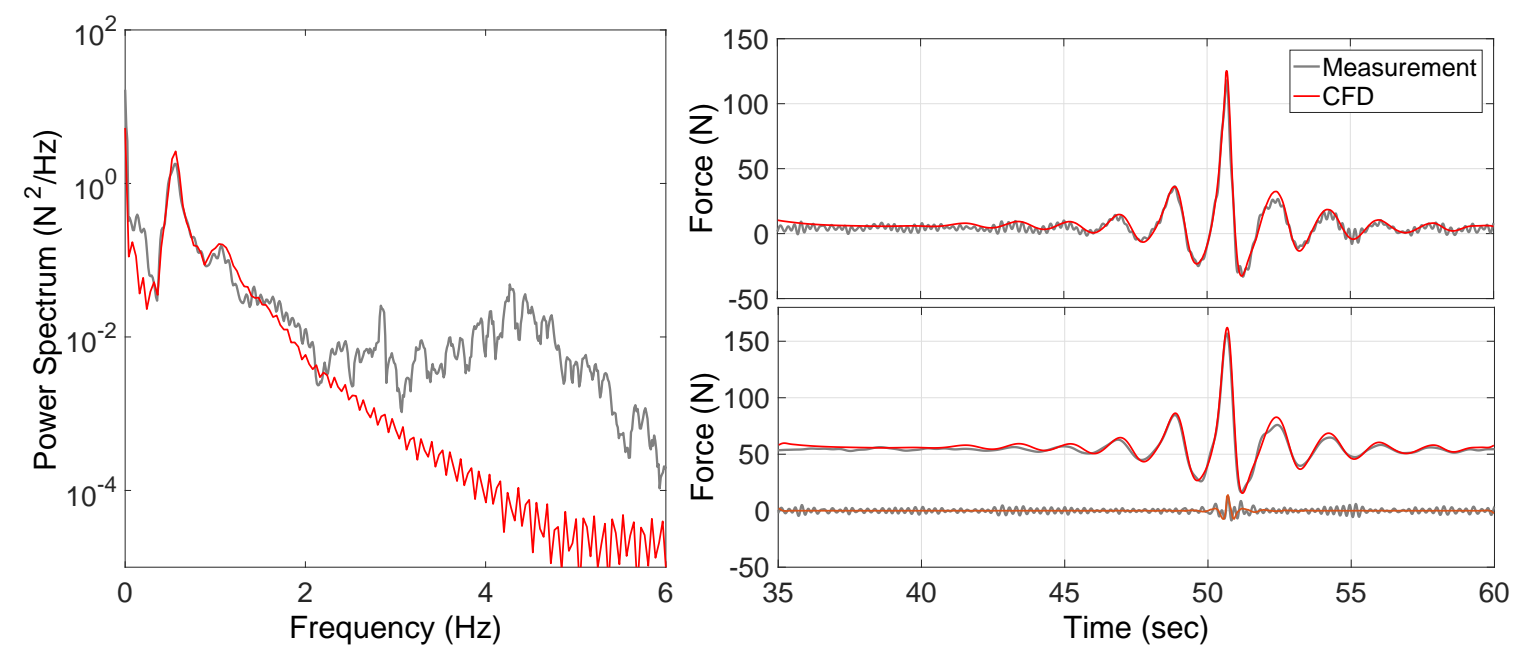

Figure 4: Comparison of measured force time histories (top right plot) for focussed wave with $0.14 \mathrm{~m} / \mathrm{s}$ current and the power spectra (left plot) between measurements (grey) and numerical predictions (red). On the bottom right plot, the total force time histories of both measurement and numerical results are split into time histories for components $\leq 2 \mathrm{~Hz}$ which reveals the dominant force components (with vertical axis shifted for clarity), and for components $>2 \mathrm{~Hz}$ which contains small high frequency components.

tion is nonlinear, producing significant $2 \mathrm{nd}(\sim 1 \mathrm{~Hz})$ and $3 \mathrm{rd}$ harmonics $(\sim 1.5 \mathrm{~Hz})$ for combined oscillatory wave velocity and steady current. These harmonics are seen in the experiments and well reproduced in the simulations. However, there is little frequency content in the CFD force spectrum beyond $2 \mathrm{~Hz}$ here, consistent with the $u|u|$ Morison form. In contrast, the experimentally measured force shows spectral contributions well above this. This is further demonstrated in Figure 4 (right plot).

The individual physical structural members are of several sizes and orientations (see Figure 2). The largest members, the main legs, have a diameter of $38.2 \mathrm{~mm}$. Hence, for the crest of the largest wave, the appropriate $K C_{l e g} \sim 40$ is sufficiently large that the steady flow value of the drag coefficient should be appropriate. The smaller structural elements have higher $K C$ values, so the steady flow drag coefficient should apply to these as well. However, at local scale, every individual structural element will have a wake. With unsteady vortex shedding, these wakes will interact and combine within and downstream of the structure in a complex manner, leading to small loading components with a broadbanded high frequency spectral tail, exactly as seen in the tank. In fact, a considerable vortex-induced-motion (VIM) of the jacket is observed during steady tow of the jacket 
model in the tank, see Figure 4, both well before and well after the passage of the wave group with a frequency centred at $\sim 4 \mathrm{~Hz}$. As soon as a finite height of wave passes through, the high frequency force components are completely swamped by the main fluid loading event.

The associated smaller-scale eddies with diameters at or larger than the individual member diameters will provide a mechanism to drive high frequency force components, as well as local turbulent flow mixing inside the porous tower (Santo et al., 2014a). The clear separation of frequency bands, here at $2 \mathrm{~Hz}$, provides strong support for our separation of length scale argument for forces. Force components below $2 \mathrm{~Hz}$ are consistent with global Morison-type loading, those above with individual structural element vortex shedding and the subsequent interactions of the small-scale eddies.

\section{Results and discussions}

In the physical tests, three towing speeds (currents) were considered: $0.14 \mathrm{~m} / \mathrm{s}$ and $0.28 \mathrm{~m} / \mathrm{s}$ (which correspond to $1.25 \mathrm{~m} / \mathrm{s}$ and $2.5 \mathrm{~m} / \mathrm{s}$, respectively, at full scale), as well as waves with no current. Using the simple blockage factor equation proposed by Taylor (1991) and subsequently adopted by American Petroleum Institute (2000), the drag coefficient for the entire jacket model, $C_{d}$, was first calibrated from the measured drag of the steady tow tests. The optimum $C_{d}$ which gives the best fit to the measured drag is found to be 1.3; high but reasonable since we do not account for local velocity amplification due to the presence of other members, in particular due to the closely-spaced conductors. This is consistent with our numerical representation of a porous tower, where there is no account for the physical volume of the structural elements within the numerical cells (no 'pore velocity representation'). This is different to the numerical representation commonly applied in modelling flow over porous coastal structures such as by Jensen et al. (2014). The overall Morison inertia coefficient, $C_{m}$, is set as 2.0, which is the potential flow-based value for cylinders in cross flow. It is worth noting that some early studies for periodic waves and current separately have demonstrated that $C_{d}$ can be larger for cylinder array than an isolated cylinder, see Cheng and Nguyen (2010); Wang et al. (2015).

These values of the Morison coefficients are used within the numerical simulation of the porous tower. As a first approximation, the density of the drag (area) and inertia (volume) of the actual jacket components is assumed to be uniformly distributed over two blocks stacked vertically and encompassing the whole volume of the porous tower. 


\subsection{Regular wave}

We first present a comparison of forces due to regular waves with and without steady current. We extract the simple periodic (steady-state) forces due to regular waves, and then decompose the total forces into drag and inertia components using the same decomposition method outlined in Santo et al. (2014b). This assumes that the drag force is in-phase with the wave crest alongside the model and that the inertia force is skew in time around this point. We subsequently phaseaverage the forces cycle-by-cycle, in a same manner as described in Santo et al. (2017). Two cases of comparison are shown in Figure 5 for regular wave of height $0.1 \mathrm{~m}$ without (top) and with 0.14 $\mathrm{m} / \mathrm{s}$ current (bottom). At field scale, these parameters become a regular wave of height $8 \mathrm{~m}$ and a current of $1.25 \mathrm{~m} / \mathrm{s}$ using Froude scaling (of 1:80 in length scales).

For each case, measurements are shown on the left, and numerical predictions on the right. The total force and the inferred drag and inertia components are plotted as solid black, blue and red lines, respectively. For the numerical predictions, solid lines are obtained using $C_{d}=1.3$ and $C_{m}=2.0$ (our default values), while the dashed lines in Figure 5 (top) are for $C_{d}=1.3 \times 1.6$ keeping the same $C_{m}$. We associate the increase in $C_{d}$ as due to Keulegen-Carpenter (KC) number effects, as previously discussed in Section 2.1. What we observe is that for all regular wave cases with both non-zero values for the in-line current, as well as for all steady tow tests with no waves, the numerical predictions using $C_{d}=1.3$ and $C_{m}=2.0$ are appropriate for reproducing all the measured force time histories. The relatively good agreement between the measurements and the numerical predictions, both in terms of the peak and trough force values but also in terms of the temporal variation of the force, provides significant support for our modelling approach.

\subsection{Focussed wave group}

We proceed with focussed wave groups with and without current, and compare our numerical prediction (accounting for wave-current blockage) with measurements taken as the reference. We also present numerical predictions according to the API recommended practice. Following the API approach, a simple blockage factor is applied to reduce the magnitude of the uniform current to account for blockage due to steady flow. The presence of the structure is assumed not to modify the undisturbed wave kinematics. The force prediction due to API is obtained numerically by integrating the undisturbed wave field and the reduced current profile over the tower volume (without the presence of the porous tower), hence it is denoted as $F_{\text {und }}$ in the captions of the 

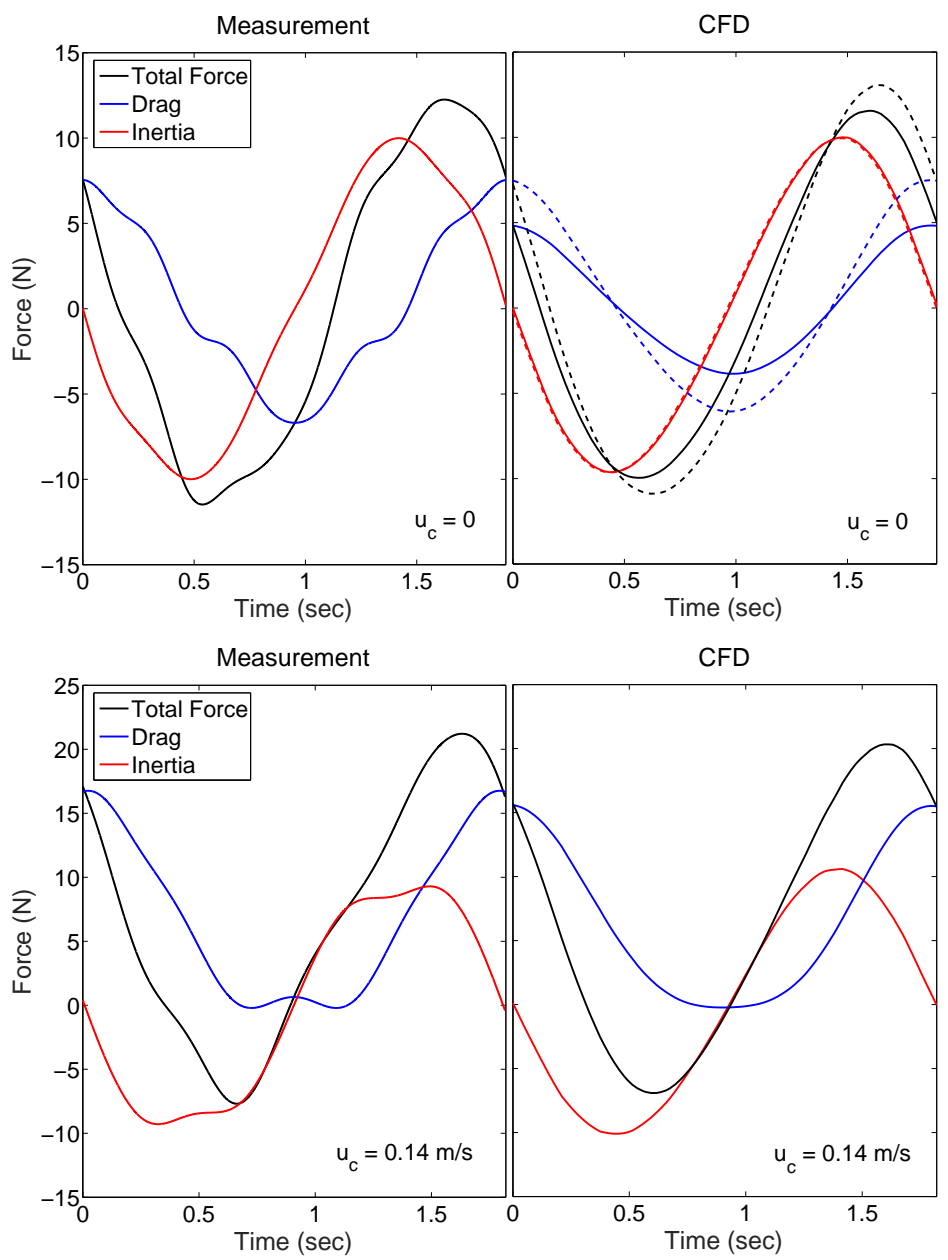

Figure 5: Comparison of force time histories for regular wave of height $0.1 \mathrm{~m}$ without current (top) and with uniform $0.14 \mathrm{~m} / \mathrm{s}$ current (bottom). The measurements are shown on the left of each subfigure, the numerical predictions on the right of each subfigure. Note the difference in vertical axis scaling between the two figures. Dashed line in top right subfigure is obtained with $C_{d}=1.3 \times 1.6$. Everywhere else we take $C_{d}=1.3$. 
following figures. On the other hand, since our numerical prediction is obtained by integrating over the tower volume by using the disturbed kinematics within the flow due to the presence of the structure as an array of obstacles, our numerical prediction is denoted as $F_{\text {dist }}$.

Figure 6 shows composite figures for comparison of surface elevation (left) and total force (right) time histories between measurements (black) and numerical predictions (grey) for focussed wave groups without current (top panel) and with a uniform $0.28 \mathrm{~m} / \mathrm{s}$ current (middle panel). The surface elevation was measured at midway between the porous tower and the side wall of the numerical wave tank. For the numerical prediction of the forces, the same values of $C_{d}=1.3$ and $C_{m}=2.0$ are used in all cases. There is no evidence of $\mathrm{KC}$ number effects for the focussed wave group without current, as previously discussed in Section 2.1. In general, reasonably good agreement in terms of surface elevation and force time histories are obtained. It is worth noting that the shape of the focussed wave group with in-line current is more compact because of the Doppler shift effect, as the modification to the wave encounter frequency was accounted for in the numerical simulation. There is a slight change in the shape of the numerical wave group due to wave-current interaction. It is also important to stress that the magnitude of the peak force is now about ten times larger than the regular wave case discussed previously. Hence, we are modelling an extreme condition, with an incoming field-scale crest elevation of $18.4 \mathrm{~m}$ (which coincidently matches the height of the infamous Draupner wave (Adcock et al., 2011) though not its likely kinematics). For a real jacket structure in the central North Sea, this would correspond to an extreme design event.

On the right middle corner of the same figure, the dashed red line represents the numerical prediction according to the API guideline, an industry design standard. Using the same $C_{d}=1.3$ and $C_{m}=2.0$, the API prediction agrees well with the measurement during the steady tow (before $t=-5 \mathrm{sec}$ ) just as the recipe is designed for. However, as the transient wave group passes by, the API recipe over-predicts the largest force when compared with the measurement (238 N reduced to $172 \mathrm{~N}$ ). In contrast, the numerical prediction using the porous tower reproduces the largest force within the correct range. The over-prediction from the API recipe is interpreted as additional blockage (by keeping the same $C_{d}$ and $C_{m}$ ) occurring within the transient waves in addition to the existing blockage due to the steady current. 

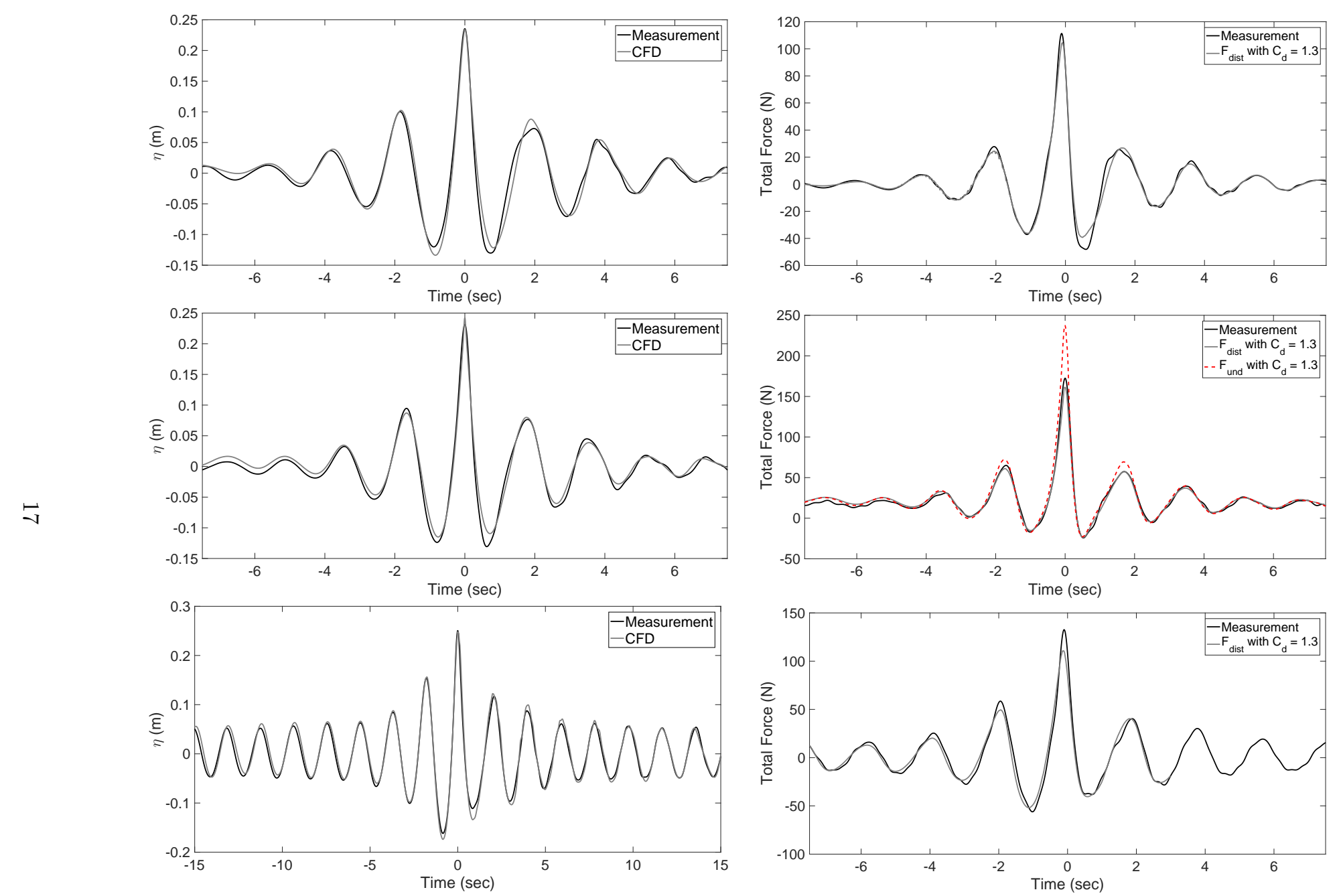

Figure 6: Comparison of surface elevation (left) and total force (right) time histories between measurements (black) and numerical predictions (grey and red) for three cases. Top panel is for a focussed wave group without current. Middle panel is for the same focussed wave group but with uniform $0.28 \mathrm{~m} / \mathrm{s}$ current. Bottom panel is for an embedded focussed wave group in $0.1 \mathrm{~m}$ regular wave background without current. 


\subsection{Embedded focussed wave in smaller regular wave background}

We proceed with the comparison of the forces arising from embedded focussed wave groups in a smaller regular wave background, with and without current. The bottom panel of Figure 6 presents the comparisons of surface elevation (left) and total force (right) time histories between measurements (black) and numerical predictions (grey and red) for the case of the embedded focussed wave group in $0.1 \mathrm{~m}$ regular wave background without current. The surface elevation is reproduced relatively well in the numerical simulation, while the total force is reasonably reproduced using $C_{d}=1.3$. It is worth remarking that comparison in terms of force components (drag and inertia) is not attempted because the resultant focussed wave group is asymmetric in time around the largest crest. This is presumably due to nonlinear wave-wave interactions occurring as the waves move down the tank to the jacket model. Hence the embedding process is noticeably distorted. Our simple force decomposition method into drag and inertia, outlined in Santo et al. (2014b), relies on symmetry around the wave crest.

Figure 7 presents comparison of embedded focussed waves in two different regular wave background heights with two different non-zero currents. Since the embedded wave group moves relative to the regular waves, the resultant wave group for the case with a $0.1 \mathrm{~m}$ regular wave background (top panel) is not perfectly focussed. Meanwhile, the wave group for the case with $0.15 \mathrm{~m}$ regular wave (middle panel) has a near-perfectly focussed deep trough. A $180^{\circ}$ phase shift to the entire input signal to the paddle for the case with a $0.15 \mathrm{~m}$ regular wave produces a wave group with a near-perfectly focussed large crest (bottom panel). Overall, the comparison in terms of surface elevation between the physical wave tank and the CFD results (left panels) is reasonable, with some slight mismatch at the adjacent crests and troughs to the largest crest/trough, due to wavecurrent interaction occurring along the numerical wave tank. The agreement around the largest crest/trough is relatively good. For the comparison in terms of force time histories (right panel), the numerical predictions with the porous tower present (grey lines) agree remarkably well with the measurements (black lines) for all cases, again using only a single set of $C_{d}=1.3$ and $C_{m}=2.0$.

The industry standard API predictions using the same $C_{d}$ and $C_{m}$ will substantially over-predict the largest force for all cases, demonstrating additional force reduction to be gained by accounting for the contribution arising from the waves. Arguably, the Morison coefficients, in particular $C_{d}$, can be tuned (i.e. in this case reduced) such that the prediction from the API recipe matches the largest peak force for each case. This is shown on the same figure as dashed red lines. For all 

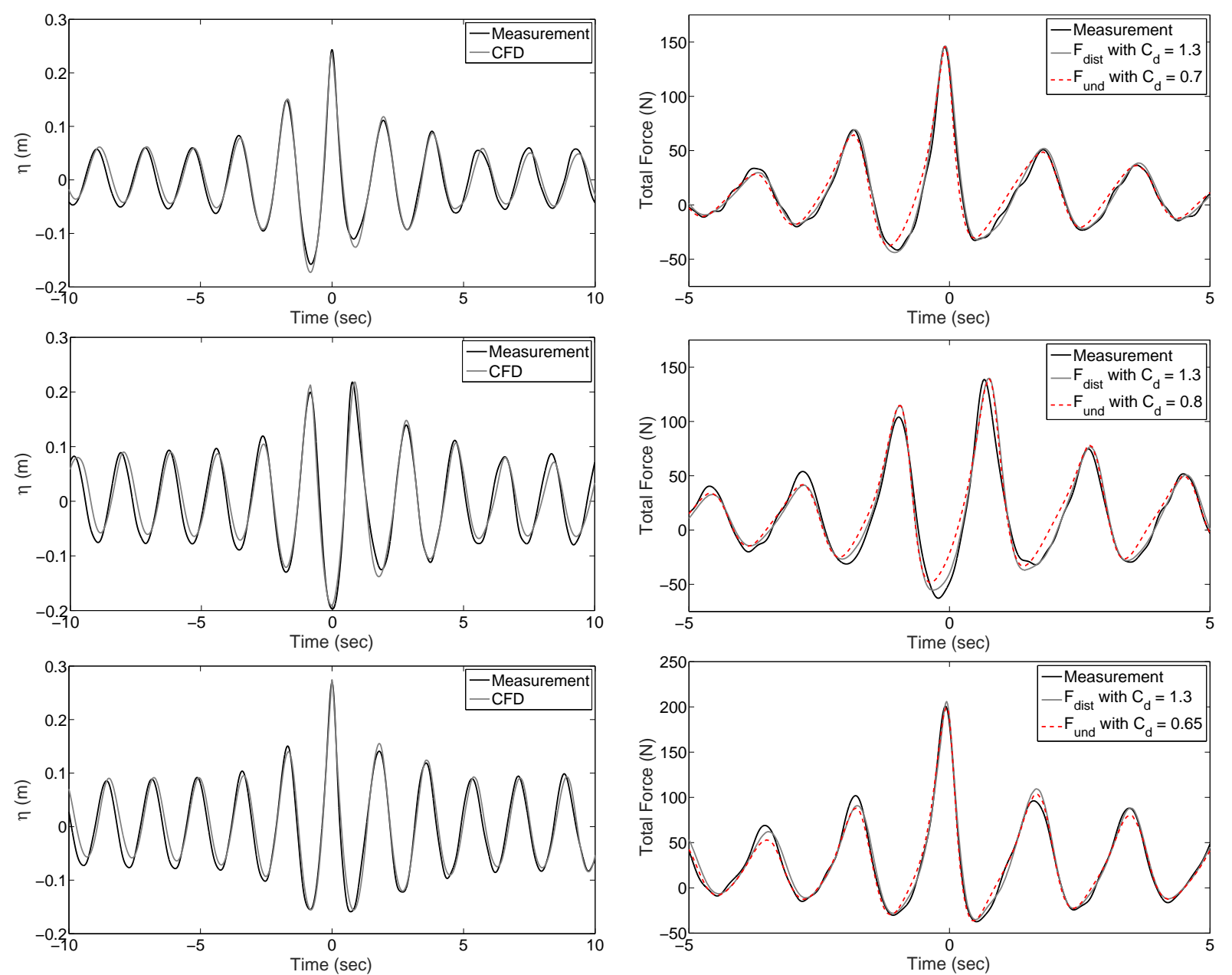

Figure 7: Comparison of surface elevation (left) and total force (right) time histories between measurements (black) and numerical predictions (grey and red) for three cases. Top panel is for an embedded focussed wave group in $0.1 \mathrm{~m}$ regular wave background with uniform $0.14 \mathrm{~m} / \mathrm{s}$ current. Middle panel is for the same embedded wave group but in $0.15 \mathrm{~m}$ regular wave with $0.14 \mathrm{~m} / \mathrm{s}$ current. Bottom panel is for a $180^{\circ}$ phase shift to embedded wave group in $0.15 \mathrm{~m}$ regular wave with $0.28 \mathrm{~m} / \mathrm{s}$ current. 
presented cases, different $C_{d}$ values (ranging from $0.65-0.8$ ) are required to match the measured largest peak forces. However, elsewhere away from the peak forces, the fit to the measured force time histories according to the API predictions are less good. Essentially, in order for the API recipe to fit the largest peak forces, one needs to use a larger $C_{d}$ for steady tow (current) only, and a set of smaller values of $C_{d}$ for waves with different heights and different in-line current speeds. Although the force comparison using the standard Morison form (which do not account for blockage effects) is not presented here, a similar trend is expected. For the Morison form to fit the largest peak forces, one needs a larger $C_{d}$ for waves only, and a set of smaller values of $C_{d}$ for current only, and waves with various in-line currents, consistent with previous observations from Allender and Petrauskas (1987) and Reed et al. (1990). Moreover, to fit the entire force time histories, one needs to use a time variant $C_{d}$.

What this paper demonstrates, is that, without the influence of $\mathrm{KC}$ number effect on $C_{d}$ (which is only present when there is regular wave field in the absence of steady current), a generality of the results can be obtained after accounting for wave-current blockage effects. A single invariant set of $C_{d}$ and $C_{m}$ is all that is required to numerically reproduce the complete measured total force time histories on a large and realistic laboratory-scale space-frame model, for a large range of combinations of various wave heights and different non-zero current speeds. This is in contrast to the results and observations of previous researchers such as Sarpkaya and Isaacson (1981), Sarpkaya et al. (1984), Heideman and Sarpkaya (1985), Rodenbusch and Källström (1986), and Chaplin et al. (1992). Many of these past studies have shown large scatter in $C_{d}$ and $C_{m}$ in particular when current is present, and none of these values bear any resemblance to those obtained under no-current condition (waves only), or steady tow condition (uniform flow at constant velocity). The observed general trend in the past is that $C_{d}$ decreases with increasing relative current velocity for a given Reynolds number and KC number. With our numerical approach using a porous tower with an embedded Morison stress field coupled with the underlying assumption of separation of length scale, no such effect is observed.

In terms of practicality, one only needs to measure the steady drag force due to steady current on a scaled or an actual space-frame offshore structure. Using the simple current blockage factor and with the information on the geometric area of the structure, the underlying $C_{d}$ can then be estimated, and $C_{m}=2.0$ appears to be a reasonable assumption for the inertia contributions. With the proposed approach, one can then obtain estimates within reasonably good accuracy of the peak 
forces as well as complete force time histories on the structure, under a wide range of extreme wave and in-line current conditions. This is particularly important when the survivability of the structure might start to come into question.

\section{Conclusions}

This paper documents laboratory-scale experimental measurements of surface elevation and total force time histories on a scaled jacket model in a large towing tank, subjected to a range of regular waves, focussed waves, and embedded focussed wave in smaller regular wave background, all with steady current present (by towing the jacket using a carriage). The quality of the measurements is demonstrated by the method of mounting the jacket, as well as the high repeatability. Accurate synchronisation between the carriage and the wave paddles allows the jacket model to meet the same focussed wave at the right location and at the right time, but with different towing speeds. Numerical simulations using CFD are conducted using a porous tower model with a uniformly distributed embedded Morison stresses representing both drag and inertia contributions to the loads on the entire jacket structure. Good agreement both in terms of surface elevation and in particular total force time histories at the model are obtained, all using a single invariant set of Morison $C_{d}=1.3$ and $C_{m}=2.0$ for large range of flow structures with non-zero different current speeds. This demonstrates the generality of the results in the absence of KC number wake-related effects.

In contrast, numerical predictions applying the present industry guidelines such as the API guidance substantially over-predict the largest peak forces using the same Morison coefficients. This is interpreted as an additional force reduction (or blockage) due to contribution from waves that is not being accounted for in the present guidelines. For the API recipe to match the peak forces, $C_{d}$ needs to be reduced to $0.65-0.8$ for large waves with steady current, and a time variant $C_{d}$ is required to match the entire total force time histories. Overall, this paper demonstrates the applicability of the porous tower modelling approach in representing a space-frame offshore structure subjected to extreme wave and current environments. Although the methodology has been tested specifically only on a particular configuration of our jacket model, we think that the general conclusions apply to other space-frame offshore structures that fall in similar Morison forcetype flow regimes. The only uncertainty is on the effects of Reynolds number on both $C_{d}$ and $C_{m}$ for turbulent flow regimes at field (prototype) scale. 
Important as these conclusions are for engineering applications, these results are equally significant from a fundamental fluid mechanics viewpoint. With the assumption of a separation of scales between the overall (global) wake of complete offshore jacket structures (several 10s of metres across at full-scale) and the wakes of individual structural members $(\sim 1-3 \mathrm{~m}$ in diameter $)$, strong wave-current-structure interaction is observed at large scale. However, there is no significant influence from small scale beyond an effectively constant value for the drag coefficient of individual structural elements. The presence of the (significant) in-line current is key for this. At physically appropriate values of the inline current used for platform design, the steady flow value for $C_{d}$ is all that is required.

These results also suggest the following hydrodynamic paradox. Consider starting with a jacket structure in significant regular waves but with no current and then increasing the current from zero. Because of the $\mathrm{KC}$ number effects, we speculate that the peak force on a jacket structure initially does not increase at all. The drag coefficient $C_{d}$ is initially affected by the coherent vortices shed from the regular waves, hence the need to amplify the value of the $C_{d}$ relative to the steady flow value. That $C_{d}$ value will subsequently drop and approach the steady flow value as the current increases over time, since the presence of current effectively washes the coherent structures downstream of the jacket model. It is thus plausible that the drop in the $C_{d}$ value in some way will balance out the increase in the force due to the same waves but with an additional current.

\section{Acknowledgements}

We thank Dr. Richard Gibson now at Offshore Consulting Group for technical advice on typical platform geometries. We are grateful for helpful discussions with Dr. Bai Wei, now of Manchester Metropolitan University, and Prof. Rodney Eatock Taylor of the University of Oxford. The technicians at Kelvin Hydrodynamics Laboratory provided a high quality facility and technical service. We would also like to acknowledge the use of the NUS High Performance Computing (HPC) facility in carrying out this work. Last but not least, we acknowledge the support from Singapore Maritime Institute (SMI) through grant number SMI-2015-AIMP-030, and the support given by Lloyds Register (LR) Foundation to the Centre for Offshore Research \& Engineering, National University of Singapore, where most of this work was carried out. LR Foundation supports the advancement of engineering-related education, and funds research and development that enhances safety of life at sea, on land and in the air. 


\section{References}

Adcock, T. A. A., Taylor, P. H., Yan, S., Ma, Q. W., Janssen, P. A. E. M., 2011. Did the Draupner wave occur in a crossing sea? Proceedings of the Royal Society A: Mathematical, Physical and Engineering Sciences 467 (2134), 3004-3021.

Allender, J., Petrauskas, C., 1987. Measured and predicted wave plus current loading on a laboratory-scale, space frame structure. In: Offshore Technology Conference, OTC 5371.

American Petroleum Institute, 2000. Recommended practice for planning, designing, and constructing fixed offshore platforms-working stress design. API RP2A-WSD 21st Edition with Erratas and Supplements 1, 130-132.

Bearman, P. W., 1967. The effect of base bleed on the flow behind a two-dimensional model with a blunt trailing edge. Aeronautical Quarterly 18, 207-224.

Berberović, E., van Hinsberg, N. P., Jakirlić, S., Roisman, I. V., Tropea, C., 2009. Drop impact onto a liquid layer of finite thickness: dynamics of the cavity evolution. Physical Review E Statistical, Nonlinear, and Soft Matter Physics 79 (3), 036306.

Chaplin, J. R., Subbiah, K., Irani, M., 1992. Local forces on a vertical cylinder in regular and irregular waves. In: The Second International Offshore and Polar Engineering Conference. International Society of Offshore and Polar Engineers.

Chen, H., Christensen, E. D., 2016. Investigations on the porous resistance coefficients for fishing net structures. Journal of Fluids and Structures 65, 76-107.

Cheng, N.-S., Nguyen, H. T., 2010. Hydraulic radius for evaluating resistance induced by simulated emergent vegetation in open-channel flows. Journal of Hydraulic Engineering 137 (9), 995-1004.

Heideman, J. C., Sarpkaya, T., 1985. Hydrodynamic forces on dense arrays of cylinders. In: Offshore Technology Conference, OTC 5008. Offshore Technology Conference.

Jacobsen, N. G., Fuhrman, D. R., Fredsøe, J., 2012. A wave generation toolbox for the open-source CFD library: OpenFOAM ${ }^{\circledR}$. International Journal for Numerical Methods in Fluids 70 (9), 1073-1088. 
Jensen, B., Jacobsen, N. G., Christensen, E. D., 2014. Investigations on the porous media equations and resistance coefficients for coastal structures. Coastal Engineering 84, 56-72.

Kristiansen, T., Faltinsen, O. M., 2012. Modelling of current loads on aquaculture net cages. Journal of Fluids and Structures 34, 218-235.

Morison, J. R., O’Brien, M. P., Johnson, J. W., Schaaf, S. A., 1950. The force exerted by surface waves on piles. Journal of Petroleum Technology 2 (5), 149-154.

Reed, K., Aarsnes, J. V., Beltrand, O., Andersen, E., 1990. Wave and current forces on conductor pipe groups. In: Environmental Forces on Offshore Structures and Their Predictions: Proceedings of an International Conference. Society of Underwater Technology.

Rodenbusch, G., Källström, C., 1986. Forces on a large cylinder in random two-dimensional flows. In: Offshore Technology Conference, OTC 5096. Offshore Technology Conference.

Santo, H., Stagonas, D., Buldakov, E., Taylor, P. H., 2017. Current blockage in sheared flow: Experiments and numerical modelling of regular waves and strongly sheared current through a space-frame structure. Journal of Fluids \& Structures 70, 374-389.

Santo, H., Taylor, P. H., Bai, W., Choo, Y. S., 2014a. Blockage effects in wave and current: 2D planar simulations of combined regular oscillations and steady flow through porous blocks. Ocean Engineering 88, 174-186.

Santo, H., Taylor, P. H., Bai, W., Choo, Y. S., 2015. Current blockage in a numerical wave tank: $3 \mathrm{D}$ simulations of regular waves and current through a porous tower. Computers \& Fluids 115, 256-269.

Santo, H., Taylor, P. H., Williamson, C. H. K., Choo, Y. S., 2014b. Current blockage experiments: force time histories on obstacle arrays in combined steady and oscillatory motion. Journal of Fluid Mechanics 739, 143-178.

Sarpkaya, T., Bakmis, C., Storm, M. A., 1984. Hydrodynamic forces from combined wave and current flow on smooth and rough circular cylinders at high Reynolds numbers. In: Offshore Technology Conference, OTC 4830. Offshore Technology Conference. 
Sarpkaya, T., Isaacson, M., 1981. Mechanics of Wave Forces on Offshore Structures. New York: Van Nostrand Reinhold.

Taylor, P. H., 1991. Current blockage: reduced forces on offshore space-frame structures. In: Offshore Technology Conference, OTC 6519.

537 Taylor, P. H., Santo, H., Choo, Y. S., 2013. Current blockage: reduced Morison forces on space frame structures with high hydrodynamic area, and in regular waves and current. Ocean Engineering $57,11-24$.

${ }_{540}$ Wang, B., Guo, X., Mei, C. C., 2015. Surface water waves over a shallow canopy. Journal of Fluid ${ }_{541}$ Mechanics 768, 572-599.

${ }_{542}$ Zhao, Y. P., Bi, C. W., Dong, G. H., Gui, F. K., Cui, Y., Guan, C. T., Xu, T. J., 2013. Numerical 543 simulation of the flow around fishing plane nets using the porous media model. Ocean Engineering $544 \quad 62,25-37$. 